\title{
A Real-World-Data Retrospective Cohort Study of Low Estrogen Receptor Positive Early Breast Cancer: Natural History and Treatment Outcomes.
}

Ricardo Costa ( $\nabla$ ricardo.costa@northwestern.edu )

H Lee Moffitt Cancer Center and Research Institute https://orcid.org/0000-0002-3303-3234

Shahla Bari

Moffitt Cancer Center

David Boulware

Moffitt Cancer Center

Jiannong Li

Moffitt Cancer Center

Loretta Loftus

Moffitt Cancer Center

Aixa Soyano

Moffitt Cancer Center

Zena Jameel

Moffitt Cancer Center

Hung Khong

Moffitt Cancer Center

Brian Czerniecki

Moffitt Cancer Center

\section{Research Article}

Keywords: Breast cancer, estrogen receptor, low-positive, recurrence free survival

Posted Date: August 12th, 2021

DOI: https://doi.org/10.21203/rs.3.rs-792365/v1

License: (c) (i) This work is licensed under a Creative Commons Attribution 4.0 International License. Read Full License 


\section{Abstract \\ Purpose}

Estrogen receptor-positive $\left(E R^{+}\right)$breast cancer $(\mathrm{BC})$ is a heterogeneous disease with an ongoing debate regarding the optimal cutoff point for clinically relevant ER expression. We used a real-world database to assess prognostic and predictive values of lower ER expression levels on treatment outcomes with endocrine therapy.

\section{Methods}

We used a nationwide electronic health record- (EHR-) derived deidentified database. Descriptive statistics were used to evaluate the correlation between ER expression, tumor characteristics, and treatment patterns among patients with early-stage BC. We used Kaplan-Meier survival curves to estimate relapse-free survival (RFS) and overall survival (OS). To assess an optimal ER expression level cut point, correlations between $\mathrm{ER}^{+}$expression and clinical outcomes were performed.

\section{Results}

Among 4697 patients with early-stage BC, 83 (1.76\%) had ER -low BC (ER expression, 1\%-9.99\%), and 36 (0.8\%) had $\mathrm{ER}^{+}$-intermediate BC (10\%-19.9\%). ER ${ }^{+}$-low tumors were associated with higher tumor grade, larger size and higher axillary tumor burden than $\mathrm{ER}^{+}$-high tumors ( $\geq 20 \%$ ER expression). African Americans had a higher prevalence of both triple-negative BC (TNBC; $21 \%)$ and $\mathrm{ER}^{+}$-low BC (22\%) than $\mathrm{ER}^{+}$-high tumors (8\%). Patients with $\mathrm{ER}^{+}$-low and $\mathrm{ER}^{+}$-intermediate tumors had survival outcomes similar to patients with TNBC and worse survival outcomes than patients with $\mathrm{ER}^{+}$-high tumors $(P<.001)$. No significant correlations between endocrine therapy and RFS or OS were observed among patients with either $\mathrm{ER}^{+}$-low or $\mathrm{ER}^{+}$-intermediate BCs. Further tumors with $<20 \%$ ER expression were associated with worse outcomes.

\section{Conclusions}

Patients with ER expression levels $<20 \%$ derive minimal benefit from endocrine therapies.

\section{Introduction}

Breast cancer (BC) is the most common malignancy worldwide. In 2020, an estimated 279100 new cases were diagnosed in the United States alone [1]. Hormone receptor-positive $\left(\mathrm{HR}^{+}\right) \mathrm{BC}$ is the most common subset ( 60\%) and is defined by the 2010 American Society of Clinical Oncology (ASCO) and College of American Pathology (CAP) guidelines as a tumor with at least $1 \%$ of cancer nuclei that stain positive for HRs for estrogen or progesterone by immunohistochemistry [2]. Notwithstanding the clinical importance of pathological assessments of both HRs, decisions pertaining to endocrine therapy (ET) are largely based on expression levels of estrogen receptors (ERs), and progesterone receptors are accepted as a prognostic biomarker.

The current definition of ER positivity presents an obvious caveat, as it does not encompass the biological heterogeneity inherent in the wide distribution of ER expression levels between tumors. Moreover, it suggests that 
patients with BCs with varying levels of ER expression have similar natural histories and derive similar benefit from approved and developing ETs. This is an incorrect premise. Data show that patients with tumors harboring lower levels of ERs have a poor prognosis similar to patients with strictly defined triple-negative BC (TNBC); patients with TNBC have a 3-year absolute risk of BC recurrence of 30\% despite multimodality therapy [3-5]. The results of a meta-analysis of 21457 patients with early-stage BC showed that ER ${ }^{+}$-low status (i.e., ligand-based assay, HR $<10$ $\mathrm{fmol} / \mathrm{mg}$ cytosol protein) was not significantly correlated with clinical benefit from adjuvant ET [6]. Current guidelines for ER testing in BCs, published by ASCO and CAP, define ER ${ }^{+}$-low tumors as cancer with 1-10\% staining for ERs and affirms the optimal cut point for ER positivity using the universally adopted immunohistochemistry scoring system [7]. It is remarkable that this revision recognizes that most of the trials supporting the benefit of adjuvant ET for early $\mathrm{ER}^{+} \mathrm{BC}$ conducted in the 1990s used quantitative measures of HRs that are no longer routinely used.

Albeit their poor prognosis and apparent lack of benefit from ETs, treatment development for patients with BCs with lower levels of ERs has been stagnant as these patients are not allowed to participate in clinical trials for patients with TNBC under the assumption of better outcomes and benefit from endocrine therapies. This is important as Developmental Therapeutics have gained momentum and continues to develop treatments targeted to strictly defined subsets of BCs (i.e., TNBC vs. ER ${ }^{+}$BCs). This is evidenced by a new surge in clinical trials that assess the efficacy of new agents targeting ERs (e.g., selective estrogen receptor modulators [SERMs] and degraders [SERDs]) [8].

We conducted a large real-world retrospective study of patients with early-stage BC to improve current understanding of the prevalence and natural history of tumors with lower levels of ER expression and treatment patterns of patients with this disease. Moreover, we retrospectively assessed the prognostic and predictive values of an alternative definition of $\mathrm{ER}^{+}$-low $\mathrm{BC}$ in relation to treatment outcomes with $\mathrm{ET}$.

\section{Methods}

\section{Data source}

This retrospective cohort study used the nationwide Flatiron Health electronic health record- (FEHR-) derived deidentified database. This is a real-world longitudinal database that comprises deidentified patient-level structured and unstructured data, which were curated via technology-enabled abstraction $[9,10]$. The database originated from approximately 280 US cancer clinics ( 800 sites of care). The majority of patients were from community oncology settings, and relative community/academic proportions may vary depending on study cohort.

Our cohort ( $\mathrm{N}=9386)$ included patients with early-stage BC diagnosed between January 2011 and May 2020. Institutional Review Board approval of the study was obtained prior to study conduct and included a waiver for informed consent. The data were deidentified and subject to obligations to prevent reidentification and protect patient confidentiality. The dataset included patient-related and tumor-related variables, including epidemiological, clinical, and pathological data as well as age at diagnosis, race, ethnicity, menopausal status, tumor size, grade, Ki67 (\%), number of lymph nodes involved, chemotherapy, and ET histories. HRs and human epidermal growth factor receptor 2 (HER2) expressions were assessed locally in accordance with standard immunohistochemistry nuclei staining by a local pathologist. Expression of HRs and HER2 was measured as the percent of nuclei staining and membrane staining, respectively. HER2 amplification was assessed according to locally assessed fluorescent in situ hybridization. 
Recurrence-free survival (RFS) was defined as the time in months from the date of first treatment to the date of diagnosis of metastasis, first local recurrence, or death, whichever occurred first; patients without these data were censored at the last date known alive. Overall survival (OS) was defined as the time from the date of first treatment to the date of death; patients without these data were censored at the last date known alive.

\section{Real-world cohort key inclusion and exclusion criteria}

Inclusion criteria used to select cases from the FEHR BC cohort included having a diagnosis of histologically proven stage $\mathrm{I}-\mathrm{II} \mathrm{BC}$ and receiving surgical treatment for BCs with curative intent. Exclusion criteria were having carcinoma in situ, metastatic $\mathrm{BC}$ and having $\mathrm{HER} 2^{+} \mathrm{BC}$. Patients who received chemotherapy before or after surgical treatment were classified as having received perioperative chemotherapy. Information on perioperative chemotherapy, ET, and

radiation therapy was abstracted. Because of the diversity in the number of chemotherapy regimens, these regimens were classified as anthracycline- and taxane-containing, anthracycline-only and taxane-only regimens, and other. Patients were followed from the date of resection (index date) until the date of biopsy-proven tumor recurrence, death, or last follow-up. Patients with $>90$ days between diagnosis and first Flatiron Health-reported structured activity were excluded to avoid missing treatment data. Furthermore, any patient with $<90$ days of follow-up from the index date was excluded.

\section{Statistical methods}

Among 9386 early-stage BC patients, 3490 had missing or incomplete ER, PR, or HER2 status and/or did not have at least 90 days of follow-up. A further 1299 patients had HER2 ${ }^{+} \mathrm{BC}$ and were also excluded from the final analysis, leaving 4697 evaluable patients (634 with TNBC and 4063 with $\mathrm{ER}^{+} \mathrm{BC}$ ).

Initially, descriptive characteristics were described by using means (standard deviations) and medians (ranges) for continuous variables and frequencies for categorical variables. The $\mathrm{ER}^{+}$-low and $\mathrm{ER}^{+}$-intermediate cohorts were compared using the Kruskal-Wallis test for continuous variables and Fisher exact test for categorical variables.

A training dataset and test dataset were established by dividing the $\mathrm{ER}^{+}$dataset into two-thirds training and onethird test data. The range of ER percent staining values was divided into 10 levels, with $10 \%$ increments. A cut point analysis was performed on the training dataset using the Contal and O'Quigley method [11], which enabled us to assess the optimum cut point of $\mathrm{ER}^{+}$staining, which was correlated with RFS using Cox proportional hazards regression. Because the analysis did not identify a cut point that was adequately and significantly differentiated from other cut points, we used $20 \%$ as a clinically reasonable cut point $(P<.0001)$. New exploratory ER ${ }^{+}$-low and $\mathrm{ER}^{+}$-high groups were defined by this cut point ( $1 \%-19.9 \%$ vs $\left.\geq 20 \%\right)$.

We then performed Kaplan-Meier survival curves for RFS and OS for both the training and test sets. Furthermore, a multivariable Cox proportional hazards survival analysis was used on the training set and adjusted for age, radiotherapy, and ET. This model was then used on the test set, and the c-statistic was used to check for model fit. Hazard ratios and 95\% confidence intervals are reported. All analyses were performed in SAS v 9.4 (Cary, NC).

\section{Results}

In the total cohort of 9386 patients with early-stage BC, only 4697 were evaluable (634 with TNBC and 4063 with $\mathrm{ER}^{+} \mathrm{BCs}$ ). Only 83 cases were classified as $\mathrm{ER}^{+}$low (ER expression 1\%-9.99\%). Median follow-up was 63 (range, 2484) months. 


\section{Baseline demographics and clinical, treatment, and outcome patterns}

The majority (72\%) of patients were White, and African Americans represented $10 \%$ of the cohort analyzed. There were 38 males in this dataset. Median age in years at diagnosis of BC was 63 (range, 24-84 years), and most women were postmenopausal (72\%). Eighty-two percent of patients had invasive ductal carcinomas, $12 \%$ had lobular carcinomas, and $47 \%$ had grade 2 tumors. Up to $63 \%$ of patients received adjuvant radiation therapy, and $37 \%$ underwent mastectomies (Table 1). Patients with $\mathrm{ER}^{+}$tumors had significantly longer RFS and OS than patients with TNBC (Fig. 1). 
Table 1

Cohort description

\begin{tabular}{|c|c|c|c|c|c|}
\hline \multirow[t]{2}{*}{ Characteristics } & & \multicolumn{4}{|l|}{ Group } \\
\hline & & $\begin{array}{l}\text { Triple negative n } \\
=634(\%)\end{array}$ & $\begin{array}{l}\mathrm{ER}^{+} \text {low n } \\
=83(\%)\end{array}$ & $\begin{array}{l}\mathrm{ER}^{+} \text {intermediate } \\
\mathrm{n}=36(\%)\end{array}$ & $\begin{array}{l}\text { ER }{ }^{+} \text {high } n= \\
3944(\%)\end{array}$ \\
\hline \multirow[t]{2}{*}{ Gender } & Female & $634(100)$ & $83(100)$ & $36(100)$ & $3906(100)$ \\
\hline & Male & $0(0)$ & $0(0)$ & $0(0)$ & $38(0.96)$ \\
\hline \multirow[t]{5}{*}{ Race } & Asian & $9(1.52)$ & $1(1.35)$ & $1(2.78)$ & 112 (3.09) \\
\hline & $\begin{array}{l}\text { Black or African } \\
\text { American }\end{array}$ & $122(20.61)$ & $16(21.62)$ & $6(16.67)$ & 288 (7.95) \\
\hline & Hispanic or Latino & $1(0.17)$ & $0(0)$ & $0(0)$ & $8(0.22)$ \\
\hline & Other & $72(12.16)$ & $7(9.46)$ & $5(13.89)$ & $549(15.16)$ \\
\hline & White & 388 (65.54) & 50 (67.57) & $24(66.67)$ & 2664 (73.57) \\
\hline \multirow[t]{2}{*}{ Ethnicity } & Hispanic or Latino & $51(8.04)$ & $7(8.43)$ & $3(8.33)$ & $247(6.26)$ \\
\hline & Non-Hispanic & $583(91.96)$ & 76 (91.57) & $33(91.67)$ & 3697 (93.74) \\
\hline \multirow[t]{5}{*}{$\begin{array}{l}\text { Menopausal } \\
\text { Status }\end{array}$} & $\begin{array}{l}\mathrm{N} / \mathrm{A} \text { - patient is } \\
\text { male }\end{array}$ & $0(0)$ & $0(0)$ & $0(0)$ & $37(0.94)$ \\
\hline & Perimenopausal & $16(2.52)$ & $1(1.2)$ & $2(5.56)$ & $95(2.41)$ \\
\hline & Postmenopausal & $436(68.77)$ & $54(65.06)$ & $24(66.67)$ & $2886(73.17)$ \\
\hline & Premenopausal & $126(19.87)$ & $21(25.3)$ & $5(13.89)$ & 691 (17.52) \\
\hline & $\begin{array}{l}\text { Unknown/not } \\
\text { documented }\end{array}$ & $56(8.83)$ & $7(8.43)$ & $5(13.89)$ & $235(5.96)$ \\
\hline \multirow[t]{4}{*}{ Histology } & Ductal & $581(91.79)$ & $75(90.36)$ & $31(86.11)$ & 3142 (79.77) \\
\hline & Lobular & $10(1.58)$ & $3(3.61)$ & $2(5.56)$ & $564(14.32)$ \\
\hline & Mixed & $9(1.42)$ & $1(1.2)$ & $1(2.78)$ & $96(2.44)$ \\
\hline & Other & $33(5.21)$ & $4(4.82)$ & $2(5.56)$ & 137 (3.48) \\
\hline \multirow[t]{3}{*}{ Tumor Grade } & Grade 1 & $10(1.58)$ & $4(5)$ & $3(8.33)$ & $1127(28.82)$ \\
\hline & Grade 2 & $113(17.91)$ & 15 (18.75) & $11(30.56)$ & $2108(53.9)$ \\
\hline & Grade 3 & $508(80.51)$ & $61(76.25)$ & $22(61.11)$ & $676(17.28)$ \\
\hline \multirow[t]{2}{*}{$\mathrm{KI}-67$} & $<20 \%$ & $28(8.95)$ & $10(25)$ & $1(6.67)$ & $1122(58.9)$ \\
\hline & $\geq 20 \%$ & 285 (91.05) & $30(75)$ & $14(93.33)$ & $783(41.1)$ \\
\hline \multirow[t]{3}{*}{ Clinical T stage } & 1 & $110(35.6)$ & $14(38.89)$ & $5(33.33)$ & 764 (59.09) \\
\hline & 2 & 135 (43.69) & $16(44.44)$ & $7(46.67)$ & 379 (29.31) \\
\hline & 3,4 & $64(20.71)$ & 6 (16.67) & $3(20)$ & $150(11.6)$ \\
\hline
\end{tabular}




\begin{tabular}{|c|c|c|c|c|c|}
\hline \multirow[t]{2}{*}{ Characteristics } & & \multicolumn{4}{|l|}{ Group } \\
\hline & & $\begin{array}{l}\text { Triple negative } \mathrm{n} \\
=634(\%)\end{array}$ & $\begin{array}{l}\text { ER }^{+} \text {low } n \\
=83(\%)\end{array}$ & $\begin{array}{l}\mathrm{ER}^{+} \text {intermediate } \\
\mathrm{n}=36(\%)\end{array}$ & $\begin{array}{l}\mathrm{ER}^{+} \text {high } \mathrm{n}= \\
3944(\%)\end{array}$ \\
\hline \multirow[t]{3}{*}{ Clinical N stage } & 0 & $160(54.98)$ & 19 (59.38) & $9(64.29)$ & 907 (76.22) \\
\hline & 1 & $100(34.36)$ & $10(31.25)$ & $4(28.57)$ & $224(18.82)$ \\
\hline & 2,3 & $31(10.65)$ & $3(9.38)$ & $1(7.14)$ & $59(4.96)$ \\
\hline \multirow[t]{2}{*}{ Radiotherapy } & No/unknown & 237 (37.38) & $29(34.94)$ & $14(38.89)$ & 1429 (36.23) \\
\hline & Yes & 397 (62.62) & $54(65.06)$ & $22(61.11)$ & 2515 (63.77) \\
\hline \multirow[t]{4}{*}{ Chemotherapy } & Other & $13(3.04)$ & $0(0)$ & $2(7.14)$ & $66(6.8)$ \\
\hline & $\begin{array}{l}\text { Anthracycline } \\
\text { without taxane }\end{array}$ & $12(2.81)$ & $0(0)$ & $3(10.71)$ & $41(4.23)$ \\
\hline & $\begin{array}{l}\text { Taxane without } \\
\text { anthracycline }\end{array}$ & $132(30.91)$ & $15(29.41)$ & $4(14.29)$ & $442(45.57)$ \\
\hline & $\begin{array}{l}\text { Taxane and } \\
\text { anthracycline }\end{array}$ & $270(63.23)$ & 36 (70.59) & $19(67.86)$ & $421(43.4)$ \\
\hline \multirow[t]{2}{*}{ Surgery } & Mastectomy & $330(54.1)$ & $42(50.6)$ & $20(55.56)$ & 2493 (64.59) \\
\hline & Other & $280(45.9)$ & $41(49.4)$ & $16(44.44)$ & 1367 (35.41) \\
\hline \multirow{2}{*}{$\begin{array}{l}\text { Endocrine } \\
\text { therapy }\end{array}$} & No & 605 (95.43) & $54(65.06)$ & $18(50)$ & $1673(42.42)$ \\
\hline & Yes & $29(4.57)$ & $29(34.94)$ & $18(50)$ & $2271(57.58)$ \\
\hline \multirow{5}{*}{$\begin{array}{l}\text { Age at } \\
\text { diagnosis }\end{array}$} & Mean & 58.5 & 55.62 & 59.53 & 62.62 \\
\hline & Median & 58.67 & 54.91 & 60.65 & 63.92 \\
\hline & Minimum & 24.03 & 26.99 & 31.05 & 25.07 \\
\hline & Maximum & 84.24 & 80.06 & 81.46 & 84.33 \\
\hline & Standard deviation & 12.35 & 13.33 & 11.66 & 11.92 \\
\hline \multirow{5}{*}{$\begin{array}{l}\text { Follow-up Time } \\
\text { (months) }\end{array}$} & Mean & 58.5 & 55.62 & 59.53 & 62.62 \\
\hline & Median & 58.67 & 54.91 & 60.65 & 63.92 \\
\hline & Minimum & 24.03 & 26.99 & 31.05 & 25.07 \\
\hline & Maximum & 84.24 & 80.06 & 81.46 & 84.33 \\
\hline & Standard deviation & 12.35 & 13.33 & 11.66 & 11.92 \\
\hline \multicolumn{6}{|c|}{$\begin{array}{l}\text { Triple negative defined as ER and } P R<1 \% \text { staining; } E R^{+} \text {low } 1 \%-9.99 \% \text { staining; } E R^{+} \text {intermediate } 10 \%-19.99 \% \text {; } \\
E^{+} \text {high } \geq 20 \% \text { staining. }\end{array}$} \\
\hline
\end{tabular}


A total of 4063 patients with $\mathrm{ER}^{+} / \mathrm{HER}^{-}$early-stage BC were included in our analysis, of whom $83(1.8 \%) \mathrm{had} \mathrm{ER}^{+}$low (ER staining, 1\%-9.99\%) and 36 (0.8\%) had ER ${ }^{+}$-intermediate (ER staining 10\%-19.99\%) BC. Both TNBC and ER ${ }^{+}$ low tumors were more commonly observed among patients $<60$ years of age; however, patients with higher ER expression were typically older. African American patients had a higher frequency of both TNBC (21\%) and ER ${ }^{+}$-low (22\%) BC than $\mathrm{ER}^{+}$-high (8\%) tumors. The majority of $\mathrm{ER}^{+}$-high tumors had low to intermediate histological grades, whereas most tumors with lower levels of ER expression were grade 3 (Table 1). Patients with ER ${ }^{+}$-high tumors were also diagnosed with smaller tumors and lower axillary tumor burden than those with $\mathrm{ER}^{+}$-low tumors.

\section{Clinical outcomes of patients with $\mathrm{ER}^{+}$-low and -intermediate early-stage BCs}

There was no statistically significant difference between the epidemiological, clinical, and pathological characteristics of patients with $\mathrm{ER}^{+}$-low and $\mathrm{ER}^{+}$-intermediate tumors (Table 2). Surgical and radiation treatment patterns were similar between these two groups. More patients in the $\mathrm{ER}^{+}$-intermediate group received adjuvant ET (unadjusted $P=.005$ ). Furthermore, more patients with $\mathrm{ER}^{+}$-low tumors received adjuvant chemotherapy with both anthracycline- and taxane-containing perioperative regimens (unadjusted, $P=.009$ ). In univariate analyses, patients with $\mathrm{ER}^{+}$-low and $\mathrm{ER}^{+}$-intermediate tumors had OS and RFS similar to patients with TNBC (Fig. 2). Because of the low number of patients in the ER-low and ER-intermediate groups, multivariate analyses were not conducted. 
Table 2

Comparison between ER low+ and ER intermediate+ groups

\begin{tabular}{|c|c|c|c|c|}
\hline \multirow[t]{2}{*}{ Characteristics } & & \multicolumn{3}{|l|}{ Group } \\
\hline & & $E R^{+}$low $n=83$ & $E R^{+}$intermediate $n=36$ & $P$ value ${ }^{*}$ \\
\hline \multirow[t]{2}{*}{ Ethnicity } & Hispanic or Latino & $7(8.43)$ & $3(8.33)$ & \multirow[t]{2}{*}{1.000} \\
\hline & Non-Hispanic & 76 (91.57) & 33 (91.67) & \\
\hline \multirow[t]{4}{*}{ Race } & Asian & $1(1.35)$ & $1(2.78)$ & \multirow[t]{4}{*}{.727} \\
\hline & Black or African American & $16(21.62)$ & $6(16.67)$ & \\
\hline & Other & $7(9.46)$ & $5(13.89)$ & \\
\hline & White & $50(67.57)$ & $24(66.67)$ & \\
\hline \multirow[t]{4}{*}{ Menopausal Status } & Perimenopausal & $1(1.2)$ & $2(5.56)$ & \multirow[t]{4}{*}{0.214} \\
\hline & Postmenopausal & $54(65.06)$ & $24(66.67)$ & \\
\hline & Premenopausal & $21(25.3)$ & $5(13.89)$ & \\
\hline & Unknown/not documented & $7(8.43)$ & $5(13.89)$ & \\
\hline \multirow[t]{4}{*}{ Histology } & Ductal & $75(90.36)$ & $31(86.11)$ & \multirow[t]{4}{*}{0.694} \\
\hline & Lobular & $3(3.61)$ & $2(5.56)$ & \\
\hline & Mixed & $1(1.2)$ & $1(2.78)$ & \\
\hline & Other & $4(4.82)$ & $2(5.56)$ & \\
\hline \multirow[t]{3}{*}{ Tumor Grade } & Grade 1 & $4(5)$ & $3(8.33)$ & \multirow[t]{3}{*}{0.229} \\
\hline & Grade 2 & 15 (18.75) & $11(30.56)$ & \\
\hline & Grade 3 & 61 (76.25) & $22(61.11)$ & \\
\hline \multirow[t]{2}{*}{ KI67 } & $<20 \%$ & $10(25)$ & $1(6.67)$ & \multirow[t]{2}{*}{0.255} \\
\hline & $>=20 \%$ & $30(75)$ & $14(93.33)$ & \\
\hline \multirow[t]{3}{*}{ Clinical T stage } & 1 & $14(38.89)$ & $5(33.33)$ & \multirow[t]{3}{*}{1.000} \\
\hline & 2 & $16(44.44)$ & $7(46.67)$ & \\
\hline & 3,4 & $6(16.67)$ & $3(20)$ & \\
\hline \multirow[t]{3}{*}{ Clinical N stage } & 0 & $19(59.38)$ & $9(64.29)$ & \multirow[t]{3}{*}{1.000} \\
\hline & 1 & $10(31.25)$ & $4(28.57)$ & \\
\hline & 2,3 & $3(9.38)$ & $1(7.14)$ & \\
\hline \multirow[t]{2}{*}{ Primary Radiotherapy } & No/unknown & $29(34.94)$ & $14(38.89)$ & \multirow[t]{2}{*}{0.684} \\
\hline & Yes & $54(65.06)$ & $22(61.11)$ & \\
\hline Surgery & mastectomy & $42(50.6)$ & $20(55.56)$ & 0.691 \\
\hline
\end{tabular}




\begin{tabular}{|c|c|c|c|c|}
\hline \multicolumn{2}{|l|}{ Characteristics } & \multicolumn{3}{|l|}{ Group } \\
\hline & & $E^{+}$low $n=83$ & $E R^{+}$intermediate $n=36$ & P value* \\
\hline & other & $41(49.4)$ & $16(44.44)$ & \\
\hline \multirow[t]{2}{*}{ Endocrine therapy } & Yes & $42(50.6)$ & $8(22.22)$ & \multirow[t]{2}{*}{0.005} \\
\hline & No & $41(49.4)$ & 28 (77.78) & \\
\hline \multirow[t]{4}{*}{ Chemotherapy } & Other & $0(0)$ & $2(7.14)$ & \multirow[t]{4}{*}{0.009} \\
\hline & Anthracycline without taxane & $0(0)$ & $3(10.71)$ & \\
\hline & Taxane without anthracycline & $15(29.41)$ & $4(14.29)$ & \\
\hline & Taxane and anthracycline & 36 (70.59) & 19 (67.86) & \\
\hline \multirow[t]{5}{*}{ Age (years) } & Mean & 55.62 & 59.53 & .130 \\
\hline & Median & 54.91 & 60.65 & \\
\hline & Minimum & 26.99 & 31.05 & \\
\hline & Maximum & 80.06 & 81.46 & \\
\hline & Standard deviation & 13.33 & 11.66 & \\
\hline \multirow[t]{5}{*}{ Follow-up (months) } & Mean & 55.62 & 59.53 & \\
\hline & Median & 54.91 & 60.65 & .130 \\
\hline & Minimum & 26.99 & 31.05 & \\
\hline & Maximum & 80.06 & 81.46 & \\
\hline & Standard deviation & 13.33 & 11.66 & \\
\hline $\begin{array}{l}\text { Abbreviations: ER, Es } \\
\text { ** ER }{ }^{+} \text {low } 1 \%-9.99 \% \\
\text { * The non-parametric } \\
\text { numerical covariates }\end{array}$ & $\begin{array}{l}\text { gen Receptor, ER } \\
\text { ning and } E R^{+} \text {intermediate } 10 \% \\
\text { value is calculated by the Krusk } \\
\text { d Fisher exact test for categoric }\end{array}$ & $\begin{array}{l}\text { 9.99\% staining. } \\
\text {-Wallis test for } \\
\text { covariates. }\end{array}$ & & \\
\hline
\end{tabular}

\section{Sensitivity analyses of ER cut point}

Kaplan-Meier survival curves for both RFS and OS showed that patients with tumors harboring ER expression levels between $1 \%$ and $19.9 \%$ had significantly poorer outcomes than patients with expression rates $\geq 20 \%$ (Fig. 3). Furthermore, a multivariable Cox proportional survival analysis was performed on the training set and showed a statistically significant correlation between higher expression of ERs ( $\geq 20 \%$ ) and improved RFS (HR, 0.55; $95 \% \mathrm{Cl}$, 0.33-0.96; $P=.02$ ) and OS (HR, 0.5; 95\% Cl, 0.27-0.9; $P=.02$ ) (Harrell's c statistics, 0.677 for RFS and 0.698 for $0 S$ ) (Table 3). Univariate analyses showed no significant treatment effect of ET on RFS and OS when comparing patients with tumor ER expression between $1 \%$ and $9.99 \%$ and $10 \%$ and $19.99 \%$ (Table 4). 
Table 3

Cox proportional hazards regression

\begin{tabular}{|c|c|c|c|c|c|c|c|c|c|}
\hline \multicolumn{10}{|c|}{ Recurrence-free survival analysis of maximum likelihood estimates } \\
\hline \multicolumn{2}{|l|}{ Parameter } & \multirow{2}{*}{ 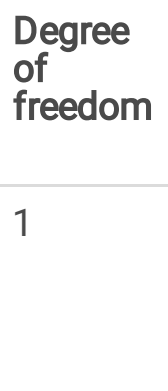 } & \multirow{2}{*}{$\begin{array}{l}\text { Parameter } \\
\text { estimate } \\
-0.59231\end{array}$} & \multirow{2}{*}{$\begin{array}{l}\text { Standard } \\
\text { error } \\
0.26311\end{array}$} & \multirow{2}{*}{$\begin{array}{l}\text { Chi- } \\
\text { Square }\end{array}$} & \multirow{2}{*}{$\begin{array}{l}P \\
\text { value } \\
.0244\end{array}$} & \multirow{2}{*}{$\begin{array}{l}\text { Hazard } \\
\text { ratio } \\
0.553\end{array}$} & \multicolumn{2}{|c|}{$\begin{array}{l}95 \% \text { Hazard } \\
\text { ratio } \\
\text { confidence } \\
\text { limits }\end{array}$} \\
\hline $\begin{array}{l}\text { *ER } \\
\text { low/Her2- }\end{array}$ & $\begin{array}{l}\text { Ref } \\
\text { ER }^{+} \\
\text {low/ } \\
\text { Her2- }\end{array}$ & & & & & & & 0.330 & 0.926 \\
\hline $\begin{array}{l}\text { Age under } \\
50\end{array}$ & $\begin{array}{l}\text { Ref } \\
\text { (50 or } \\
\text { above) }\end{array}$ & 1 & -0.14587 & 0.15137 & 0.9286 & .3352 & 0.864 & 0.642 & 1.163 \\
\hline $\begin{array}{l}\text { Endocrine } \\
\text { therapy }\end{array}$ & $\begin{array}{l}\text { Ref } \\
\text { (no) }\end{array}$ & 1 & -0.89719 & 0.17325 & 26.8186 & $<.0001$ & 0.408 & 0.290 & 0.573 \\
\hline $\begin{array}{l}\text { Primary } \\
\text { radiotherapy }\end{array}$ & $\begin{array}{l}\text { Ref } \\
\text { (no) }\end{array}$ & 1 & -0.55534 & 0.11269 & 24.2846 & $<.0001$ & 0.574 & 0.460 & 0.716 \\
\hline \multicolumn{10}{|c|}{ Fitting model to the test set, at 3 years recurrence-free survival, Harrell's c-statistic $=0.677$. } \\
\hline \multicolumn{10}{|c|}{ Overall Survival Analysis of Maximum Likelihood Estimates } \\
\hline $\begin{array}{l}\mathrm{ER}^{+} \\
\text {High/Her2 }\end{array}$ & $\begin{array}{l}\text { Ref } \\
\text { (ER } \\
\text { low/ } \\
\text { Her2-) }\end{array}$ & 1 & -0.69144 & 0.30380 & 5.1799 & .0228 & 0.501 & 0.276 & 0.908 \\
\hline $\begin{array}{l}\text { Age under } \\
50\end{array}$ & $\begin{array}{l}\text { Ref } \\
\text { (50 or } \\
\text { above) }\end{array}$ & 1 & -0.60046 & 0.20565 & 8.5258 & .0035 & 0.549 & 0.367 & 0.821 \\
\hline $\begin{array}{l}\text { Endocrine } \\
\text { therapy }\end{array}$ & $\begin{array}{l}\text { Ref } \\
\text { (no) }\end{array}$ & 1 & -0.79007 & 0.20488 & 14.8702 & .0001 & 0.454 & 0.304 & 0.678 \\
\hline $\begin{array}{l}\text { Primary } \\
\text { radiotherapy }\end{array}$ & $\begin{array}{l}\text { Ref } \\
\text { (no) }\end{array}$ & 1 & -0.63333 & 0.13218 & 22.9577 & $<.0001$ & 0.531 & 0.410 & 0.688 \\
\hline \multicolumn{10}{|c|}{ Fitting model to the test set, at 3 years overall survival, Harrell's c-statistic $=0.698$. } \\
\hline \multicolumn{10}{|c|}{ Abbreviations: $\mathrm{Cl}$, confidence interval, $\mathrm{Cl}$; ER, estrogen receptor; Ref, reference. } \\
\hline
\end{tabular}


Table 4

Endocrine therapy treatment effect (univariate analysis)

\begin{tabular}{|c|c|c|c|c|c|c|c|}
\hline \multirow[b]{2}{*}{ Parameter } & \multirow[b]{2}{*}{$\begin{array}{l}\text { Patients, } \\
\text { no. }\end{array}$} & \multicolumn{3}{|c|}{ Recurrence free survival } & \multicolumn{3}{|c|}{ Overall survival } \\
\hline & & $\begin{array}{l}\text { Events, } \\
\text { no. }\end{array}$ & $\mathrm{HR}(95 \% \mathrm{Cl})$ & $\begin{array}{l}P \\
\text { value }\end{array}$ & $\begin{array}{l}\text { Events, } \\
\text { no. }\end{array}$ & $\mathrm{HR}(95 \% \mathrm{Cl})$ & $\begin{array}{l}P \\
\text { value }\end{array}$ \\
\hline \multicolumn{8}{|c|}{ Patient who received ET } \\
\hline ER low (1-9.99\%) & 41 & 5 & Ref & Ref & 3 & Ref & Ref \\
\hline $\begin{array}{l}\text { ER intermediate (10- } \\
20 \%)\end{array}$ & 28 & 5 & $\begin{array}{l}1.47(0.42 \\
5.08)\end{array}$ & .545 & 5 & $\begin{array}{l}2.57(0.61 \\
10.77)\end{array}$ & .196 \\
\hline \multicolumn{8}{|l|}{$\begin{array}{l}\text { Patient who did not } \\
\text { receive ET }\end{array}$} \\
\hline ER low (1-9.99\%) & 42 & 16 & Ref & Ref & 10 & Ref & Ref \\
\hline $\begin{array}{l}\text { ER intermediate (10- } \\
20 \%)\end{array}$ & 8 & 2 & $\begin{array}{l}0.38 \\
(0.05,2.86)\end{array}$ & .346 & 1 & $\mathrm{n} / \mathrm{a}$ & $\mathrm{n} / \mathrm{a}$ \\
\hline
\end{tabular}

\section{Discussion}

$\mathrm{ER}^{+} \mathrm{BC}$ is a heterogenous disease, not only in its natural history but also in reference to its wide distribution of ER expression levels between tumors; cases of low expression represent a small proportion of cases of BC. The optimal cut point that indicates reliable prognostic ER expression and predictive information of ET benefit remains to be determined. Historically, any level of ER expression has been considered to be positive, which is counterintuitive and misleading, as tumors harboring lower levels of ERs will likely not respond to ET. In parallel, tumors with low expression rates of ERs are universally excluded from contemporary clinical trials for patients with TNBC. The corollary is that the $\mathrm{ER}^{+}$-low BC subset remains an orphan disease. With an increasing number of clinical trials aiming to develop new ETs (e.g., SERDs and SERMs), a better understanding of the natural history of and possible benefit from ET remains an unmet need in developmental therapeutics for patients with ER ${ }^{+}-$low BCs.

We conducted a large real-world retrospective cohort study of 4697 participants with early-stage $\mathrm{ER}^{+} \mathrm{BC}$ or $\mathrm{TNBC}^{\text {In }}$ this cohort, the natural history of patiets with eraly stage BCs was similar to previously published literature [12]. Our results suggest that $E R^{+}$-low $B C$ (expression, 1\%-9.99\%) represents a small subset (1.8\%) of patients with $E R^{+} B C$. These tumors presented pathological features and prognosis similar to those of patients with TNBC. We retrospectively assessed a new cut point for the definition of $\mathrm{ER}^{+}$-low $\mathrm{BC}$. Patients with tumors harboring ER expression rates between $10 \%$ and $19.9 \%$ (intermediate levels) presented similar clinicopathological features, natural histories, and treatment patterns to those with ER ${ }^{+}$-low BCs (1\%-9.99\%); however, anthracycline- and taxanecontaining chemotherapy regimens were more commonly administered to patients with tumors with ER expression levels $<10 \%$. Moreover, ET was not associated with clinically meaningful improvement of RFS and OS among either patients with 1-9.99\% ER expression or patients with 10-20\% ER expression. We also observed that an incremental expression of ER is associated with improved outcomes, further suggesting that the binary categorization of ER expression represents a simplification of continuous biological phenomena. 
Our study has limitations inherent to retrospective cohort studies. There were missing data, which prevented us from including important variables, such as chemotherapy and nodal staging, into our adjusted analyses. Assessments of ER expression were not centrally reviewed. Additionally, the number of ER ${ }^{+}$-low and $\mathrm{ER}^{+}$-intermediate tumors was relatively small compared to other subtypes, limiting exploratory statistical comparisons and multivariable adjustments. Nonetheless, this is a large retrospective study that uses real-world data, which thereby increases the external validity of our findings. Given the low frequency of $E R^{+}-$low tumors, the conduction of large prospective studies assessing the prognostic and predictive value of lower ER expression is practically unfeasible. This study therefore fills an important gap in our knowledge of the natural history of ER ${ }^{+}$-low and $\mathrm{ER}^{+}$-intermediate tumors.

\section{Conclusions}

Findings from this real-world retrospective study suggest that patients with ER expression rates $<20 \%$ have poor outcomes and derive minimal benefit from ETs. Future clinical trials for patients with TNBC should allow for participation of patients with ER-low BCs.

\section{Declarations}

Funding: Shula's Foundation.

Ethical Approval: Institutional Review Board approval of the study protocol was obtained from WCG IRB prior to study conduct and included a waiver of informed consent.

Informed Consent: This article does not contain any studies with human participants which would require informed consent.

Availability of data and materials: The data that support the findings of this study have been originated by Flatiron Health, Inc. These de-identified data may be available upon request and are subject to a license agreement with Flatiron Health; interested researchers should contact DataAccess@flatiron.com to determine licensing terms.

Acknowledgements: Editorial assistance was provided by the Moffitt Cancer Center's Office of Scientific Writing by Dr. Paul Fletcher, Daley Drucker, and Mikael Michalik. No compensation was given beyond their regular salaries. This work has been supported in part by the Biostatistics \& Bioinformatics Shared Resource at the H. Lee Moffitt Cancer Center \& Research Institute, a comprehensive cancer center designated by the National Cancer Institute and funded in part by Moffitt's Cancer Center Support Grant (P30-CA076292)

Conflicts of Interest: R. L. B. Costa received honorarium from Bristol Meyers Squib, Pfizer, Athenex Oncology, Daiichi Sankyo and Astra Zeneca. All other authors declare no conflicts of interest.

\section{References}

1. Siegel RL, Miller KD, Jemal A (2020) Cancer statistics, 2020. Cancer J Clin 70:7-30. doi:10.3322/caac.21590

2. Hammond ME, Hayes DF, Dowsett M, Allred DC, Hagerty KL, Badve S, Fitzgibbons PL, Francis G, Goldstein NS, Hayes M, Hicks DG, Lester S, Love R, Mangu PB, McShane L, Miller K, Osborne CK, Paik S, Perlmutter J, Rhodes A, Sasano H, Schwartz JN, Sweep FC, Taube S, Torlakovic EE, Valenstein P, Viale G, Visscher D, Wheeler T, Williams RB, Wittliff JL, Wolff AC (2010) American Society of Clinical Oncology/College Of American 
Pathologists guideline recommendations for immunohistochemical testing of estrogen and progesterone receptors in breast cancer. J Clin Oncol 28:2784-2795. doi:10.1200/JC0.2009.25.6529

3. Landmann A, Farrugia DJ, Zhu L, Diego EJ, Johnson RR, Soran A, Dabbs DJ, Clark BZ, Puhalla SL, Jankowitz RC, Brufsky AM, Ahrendt GM, McAuliffe PF, Bhargava R (2018) Low Estrogen Receptor (ER)-Positive Breast Cancer and Neoadjuvant Systemic Chemotherapy: Is Response Similar to Typical ER-Positive or ER-Negative Disease? Am J Clin Pathol 150:34-42. doi:10.1093/ajcp/aqy028

4. Prabhu JS, Korlimarla A, Desai K, Alexander A, Raghavan R, Anupama C, Dendukuri N, Manjunath S, Correa M, Raman N, Kalamdani A, Prasad M, Gopinath KS, Srinath BS, Sridhar TS (2014) A Majority of Low (1-10\%) ER Positive Breast Cancers Behave Like Hormone Receptor Negative Tumors. J Cancer 5:156-165. doi:10.7150/jca.7668

5. Raghav KP, Hernandez-Aya LF, Lei X, Chavez-Macgregor M, Meric-Bernstam F, Buchholz TA, Sahin A, Do KA, Hortobagyi GN, Gonzalez-Angulo AM (2012) Impact of low estrogen/progesterone receptor expression on survival outcomes in breast cancers previously classified as triple negative breast cancers. Cancer 118:14981506. doi:10.1002/cncr.26431

6. Davies C, Godwin J, Gray R, Clarke M, Cutter D, Darby S, McGale P, Pan HC, Taylor C, Wang YC, Dowsett M, Ingle $J$, Peto R (2011) Relevance of breast cancer hormone receptors and other factors to the efficacy of adjuvant tamoxifen: patient-level meta-analysis of randomised trials. Lancet 378:771-784. doi:10.1016/s01406736(11)60993-8

7. Allison KH, Hammond MEH, Dowsett M, McKernin SE, Carey LA, Fitzgibbons PL, Hayes DF, Lakhani SR, ChavezMacGregor M, Perlmutter J, Perou CM, Regan MM, Rimm DL, Symmans WF, Torlakovic EE, Varella L, Viale G, Weisberg TF, McShane LM, Wolff AC (2020) Estrogen and Progesterone Receptor Testing in Breast Cancer: ASCO/CAP Guideline Update. Journal of clinical oncology: official journal of the American Society of Clinical Oncology 38:1346-1366. doi:10.1200/jco.19.02309

8. Howell SJ, Johnston SR, Howell A (2004) The use of selective estrogen receptor modulators and selective estrogen receptor down-regulators in breast cancer. Best Pract Res Clin Endocrinol Metab 18:47-66. doi:10.1016/j.beem.2003.08.002

9. Ma X, Long L, Moon S, Adamson BJS, Baxi SS (2020) Comparison of Population Characteristics in Real-World Clinical Oncology Databases in the US: Flatiron Health, SEER, and NPCR. medRxiv:2020.2003.2016.20037143. doi: 10.1101/2020.03.16.20037143

10. Benjamin Birnbaum NN, Katharina Seidl-Rathkopf M, Agrawal M, Estevez E, Estola J, Haimson L, He P, Larson P, Richardson (2020) Model-assisted cohort selection with bias analysis for generating large-scale cohorts from the EHR for oncology research. In

11. Tunes-da-Silva G, Klein JP (2011) Cutpoint selection for discretizing a continuous covariate for generalized estimating equations. Comput Stat Data Anal 55:226-235. doi:10.1016/j.csda.2010.02.016

12. Liedtke C, Mazouni C, Hess KR, Andre F, Tordai A, Mejia JA, Symmans WF, Gonzalez-Angulo AM, Hennessy B, Green M, Cristofanilli M, Hortobagyi GN, Pusztai L (2008) Response to neoadjuvant therapy and long-term survival in patients with triple-negative breast cancer. J Clin Oncol 26:1275-1281.

doi:10.1200/JCO.2007.14.4147

\section{Figures}

Page $14 / 16$ 
A. Recurrence free survival

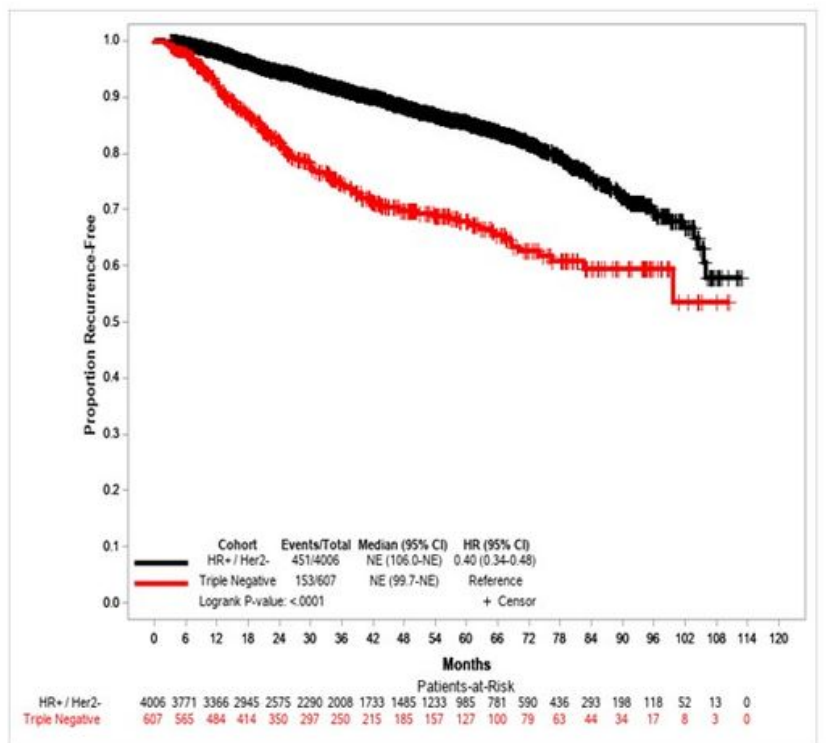

B. Overall survival

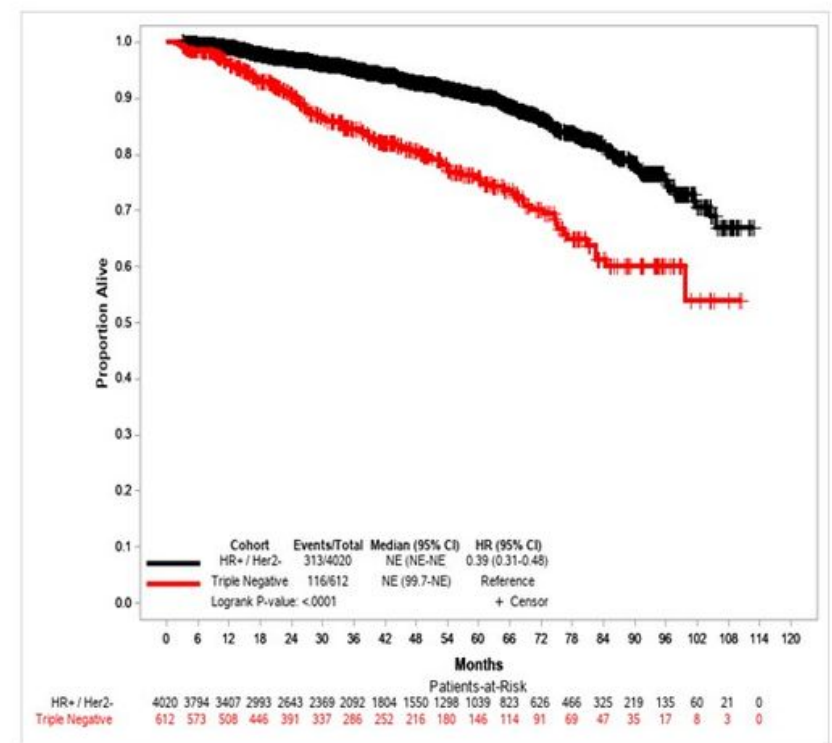

\section{Figure 1}

Clinical outcomes of patients with ER+ and TNBC BCs. Triple negative defined as ER/PR $<1 \%$ staining and HER2(i.e., IHC 0/1+ and non-amplified); ER+ $\geq 1 \%$ staining. Abbreviations: Breast cancer, BC; Estrogen Receptor, ER; Human Epidermal growth factor receptor 2, HER2; immunohistochemistry (IHC).

A. Recurrence free survival

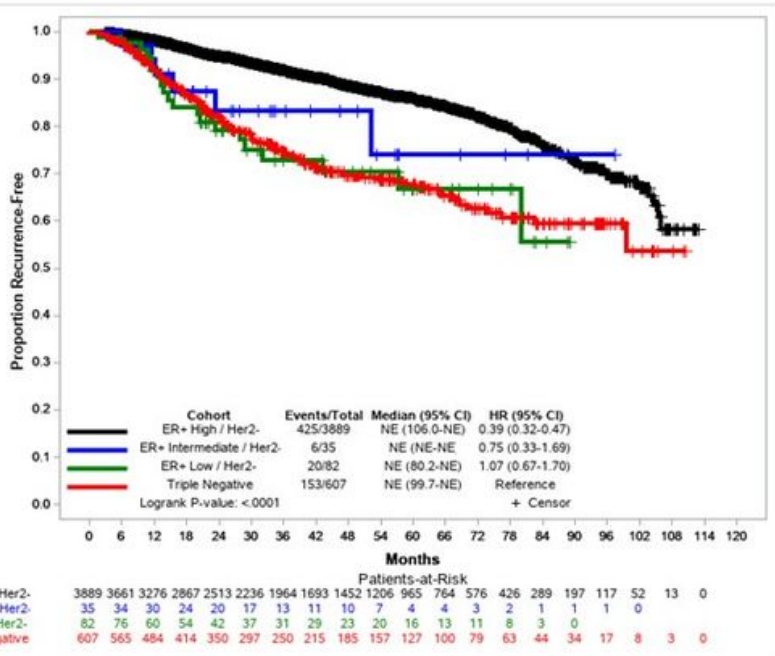

B. Overall survival

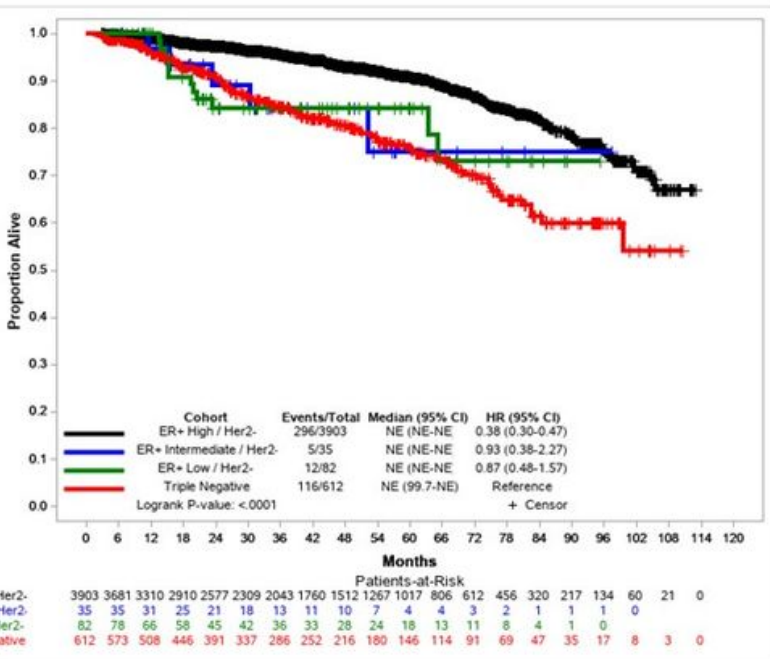




\section{Figure 2}

Clinical outcomes of patients with TN, low-, intermediate- and High-ER+ BCs. Triple negative defined as ER and PR < $1 \%$ staining, ER+ low 1-9.99\% staining, ER+ inter, 10-19.99\%, ER+ high $\geq 20 \%$ staining. Abbreviations: Breast cancer, BC; Estrogen Receptor, ER; Human Epidermal growth factor receptor 2, HER2; triple negative, TN.

A. Recurrence free survival - Training set

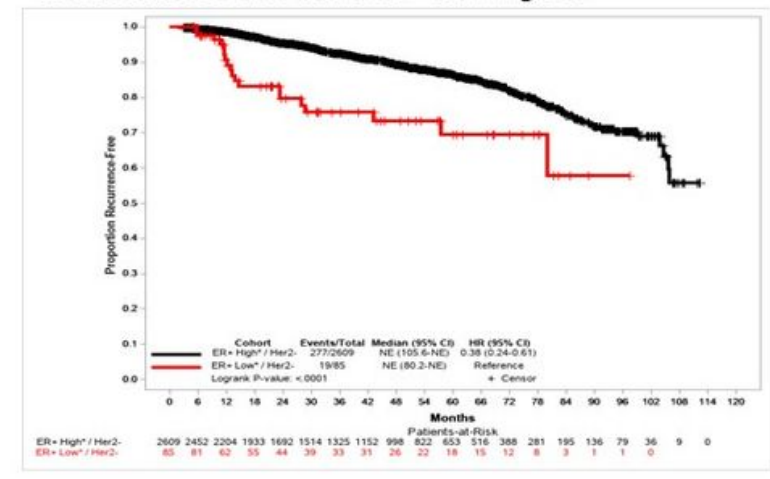

C. Overall survival - Training set

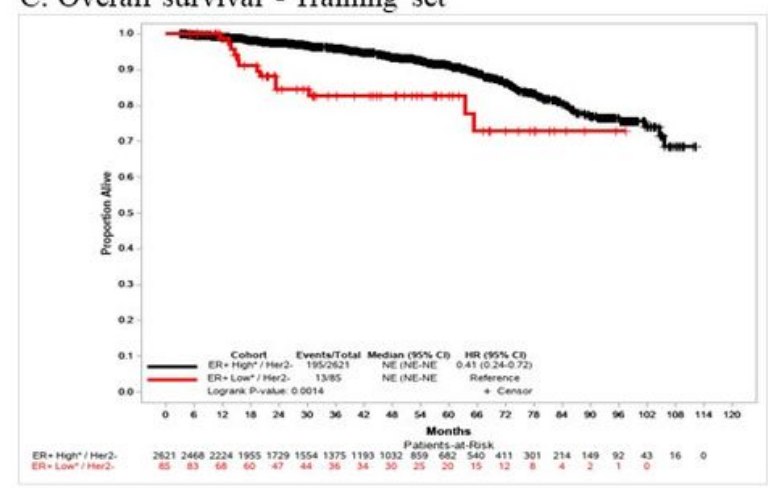

B. Recurrence free survival- test set

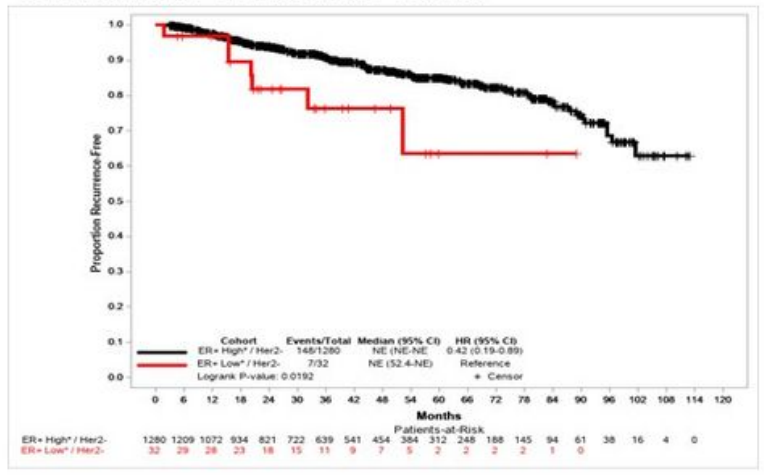

D. Overall survival - test set

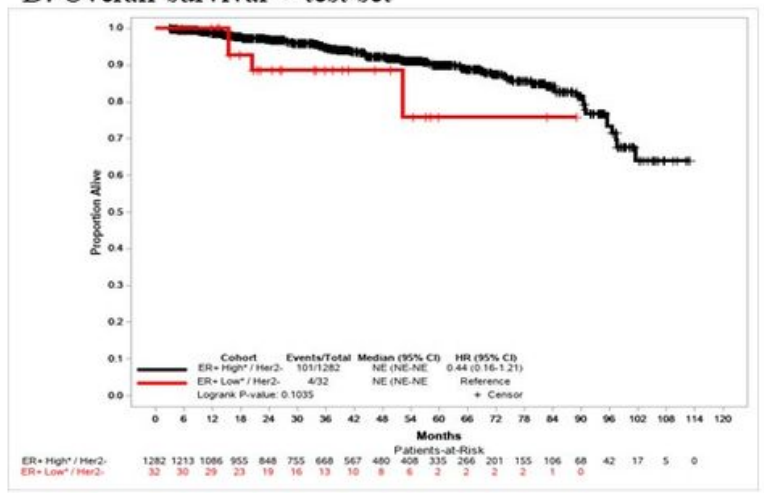

\section{Figure 3}

Clinical outcomes of patients with ER low positive vs. ER high positive BCs. ER+ low 1-19.9\% staining and ER+ high $\geq 20 \%$ staining. Abbreviations: Breast cancer, BC; Estrogen Receptor, ER. 